\title{
Specialist geriatric medical assessment for patients discharged from hospital acute assessment units: randomised controlled trial
}

\author{
(c) (1) (8) OPEN ACCESS
}

\author{
Judi Edmans senior research fellow ${ }^{1}$, Lucy Bradshaw medical statistician ${ }^{1}$, Matthew Franklin health \\ economist $^{2}$, John Gladman professor of medicine for older people ${ }^{1}$, Simon Conroy \\ geriatrician/honorary senior lecturer ${ }^{3}$
}

'University of Nottingham, Division of Rehabilitation and Ageing, Medical School, Queens Medical Centre, Nottingham NG7 2UH, UK; ${ }^{2}$ University of Nottingham, Division for Social Research in Medicines and Health, Medical School, Queens Medical Centre, Nottingham NG7 2UH, UK; ${ }^{3}$ University Hospitals of Leicester, Room 034, Level 5, Windsor Building, Leicester Royal Infirmary, Leicester LE1 5WW, UK

\begin{abstract}
Objective To evaluate the effect of specialist geriatric medical management on the outcomes of at risk older people discharged from acute medical assessment units.

Design Individual patient randomised controlled trial comparing intervention with usual care.

Setting Two hospitals in Nottingham and Leicester, UK.

Participants 433 patients aged 70 or over who were discharged within 72 hours of attending an acute medical assessment unit and at risk of decline as indicated by a score of at least 2 on the Identification of Seniors At Risk tool.

Intervention Assessment made on the acute medical assessment unit and further outpatient management by specialist physicians in geriatric medicine, including advice and support to primary care services.

Main outcome measures The primary outcome was the number of days spent at home (for those admitted from home) or days spent in the same care home (if admitted from a care home) in the 90 days after randomisation. Secondary outcomes were determined at 90 days and included mortality, institutionalisation, dependency, mental wellbeing, quality of life, and health and social care resource use.
\end{abstract}

Results The two groups were well matched for baseline characteristics, and withdrawal rates were similar in both groups (5\%). Mean days at home over 90 days' follow-up were 80.2 days in the control group and 79.7 in the intervention group. The $95 \%$ confidence interval for the difference in means was -4.6 to 3.6 days $(P=0.31)$. No significant differences were found for any of the secondary outcomes.

Conclusions This specialist geriatric medical intervention applied to an at risk population of older people attending and being discharged from acute medical units had no effect on patients' outcomes or subsequent use of secondary care or long term care.

\section{Introduction}

Many acute hospitals across the Western world operate a system whereby patients presenting as an emergency are assessed in an acute assessment area. In the United Kingdom, such facilities are commonly referred to as acute medical units. ${ }^{1}$ Many patients can be discharged home from acute medical units after assessment or a short period of stabilisation. Poor outcomes and high resource use are common in older people presenting to acute medical units who are discharged directly: in one UK series, $58 \%$ subsequently re-presented and $29 \%$ died over the next year ${ }^{2}$; in another, $76 \%$ had one or more adverse outcomes (death institutionalisation, readmission, increase in dependency, or decline in mental wellbeing or quality of life) over three months. ${ }^{3}$

Such poor outcomes may be avoidable. An unpublished local audit reviewing the medical care of 50 frail patients on one acute medical unit identified one or more additional and potentially valuable interventions that could be provided to $80 \%$ of patients, including drug reviews, referral to community services, falls management, capacity assessments, end of life care, and the medical treatment of otherwise missed conditions. Inappropriate prescribing (49\% $v 27 \%$ ) and under-prescribing of appropriate drugs $(30 \% v 15 \%)$ are common in acute care settings. ${ }^{4}$

These observations have led to consideration of a specialist geriatric medical intervention for at risk older people on acute medical units to reduce the incidence of adverse outcomes and associated high resource use. A new service was developed in the English East Midlands, in which frail older patients being discharged from acute medical units were assessed by geriatricians, who also coordinated short term community follow-up to continue the assessment and oversaw the delivery 
of medical and non-medical community interventions. ${ }^{5}$ This has been referred to as "interface geriatrics." ${ }^{.5-9} \mathrm{We}$ did a randomised controlled trial to evaluate whether additional specialist geriatric medical expertise (interface geriatrics) aiming to overcome deficiencies in routine medical care on acute medical units could improve patients' outcomes. We proposed that a successful intervention would be indicated by an increase in the number of days of follow-up that patients spent living in their own at homes and a corresponding decrease in the number of days that patients spent in health or social care institutions (such as hospitals and care homes).

\section{Methods \\ Design}

We did a one to one parallel group individual patient randomised controlled trial in two hospitals in Nottingham (catchment population 675 000) and Leicestershire (catchment population 1.1 million), East Midlands, UK. The trial protocol has been published. ${ }^{10}$

\section{Participants}

Patients were eligible if they were discharged from an acute medical unit within 72 hours of attending hospital, were aged 70 or over, and were identified as being at heightened risk of future health problems (defined by a score of at least $2 / 6$ on the Identification of Seniors At Risk tool ${ }^{11-13}$ ). Exclusion criteria were not being resident in the hospital catchment area, lacking mental capacity to give informed consent and without a consultee, any exceptional reason cited by acute medical unit staff why patients should not be recruited, and participation in other related studies.

\section{Recruitment}

Trained researchers embedded in the acute medical units recruited participants. Potential participants with the mental capacity to do so and who agreed gave written consent. A family caregiver was asked to act a consultee for potential participants lacking capacity. If no family caregiver was available, the medical practitioner on the acute medical unit responsible for their care was asked to act as a consultee.

\section{Baseline data}

Baseline data for participants comprised demographics (age, sex, ethnicity, marital status, residential status, education, and social and financial questions), Identification of Seniors At Risk score, health conditions (presenting problems, comorbidities (Charlson co-morbidity index $)^{14}$ and list of drugs, cognitive function (Folstein Mini-Mental State Examination, ${ }^{15}{ }^{16}$ personal activities of daily living (Barthel ADL Index), ${ }^{17}$ health related quality of life/status (EuroQoL EQ-5D and ICEpop CAPability measure for older people (ICECAP-O), ${ }^{18}{ }^{19}$ and psychological wellbeing (General Health Questionnaire 12). ${ }^{20}$

\section{Randomisation}

We used a secure internet based system to randomise individual participants one to one between the intervention and control groups, with stratification by centre. The Nottingham Clinical Trials Support Unit (http://ctu.nottingham.ac.uk/ctu/) created the randomisation sequence by using random permuted blocks of randomly varying size. After gaining patients' consent, researchers logged into a remote, internet based randomisation system to access the randomised treatment allocation. The nature of the intervention meant that blinding participants or services to the allocation was not possible.

\section{Interventions}

Usual care on the acute medical units before recruitment for both the control and intervention groups comprised assessment and treatment by a consultant physician and attending medical team. Some patients were referred to a multidisciplinary team (physiotherapist, occupational therapist, nurse). Patients' general practitioners were responsible for all aftercare. Participants in the control group received no additional intervention over and above usual care.

Participants in the intervention group were assessed before discharge from the acute medical unit by one of 12 geriatricians (either senior trainees or fully qualified specialists), who aimed to coordinate the delivery of whatever additional immediate care or aftercare they deemed necessary. Such care could include a review of diagnoses; a drug review; further assessment at home or in a clinic or by recommending admission rather than discharge; advance care planning; or liaison with primary care, intermediate care, and specialist community services. The intervention was expected to be complete within one month of randomisation. Further details of the intervention are described elsewhere. ${ }^{5}$ All study geriatricians completed logs of their intervention, which included the initial assessment, location this took place, and its duration; the interval from initial assessment to next visit; the number and duration of follow-up home visits and phone calls; the number of clinic visits arranged; other patient related activities and their duration; and free text to list key additional interventions.

\section{Outcomes}

The primary outcome was the number of days spent at home in the 90 days after randomisation. ${ }^{21}$ This composite outcome took account of death, time spent in hospital, and any new care home placements. Secondary outcomes were ascertained at 90 days and comprised death, institutionalisation, secondary care contacts (number of hospital presentations, defined as the total number of inpatient admissions, attendances to accident and emergency/acute medical unit without admission, and day cases during the 90 day follow-up period), dependency in personal activities of daily living (Barthel ADL), self reported falls, psychological wellbeing (General Health Questionnaire 12) and health related quality of life (EQ-5D and ICECAP-O).

\section{Data collection}

The embedded research staff collected baseline data at an interview. Research staff (blind to allocation) determined outcomes, checked hospital and general practice records for deaths and changes of address, and administered postal questionnaires. We obtained secondary care resource use by using electronic extraction from routine databases covering acute and sub-acute hospitals in the two areas.

\section{Statistical analysis}

We used the intention to treat principle to analyse data according to a pre-specified plan. We compared the number of days spent at home in each group by using the non-parametric van Elteren's test stratified by centre (a generalisation of the Mann-Whitney test), using bootstrapping to calculate a $95 \%$ confidence interval for the difference in the mean number of days at home. We analysed secondary outcomes by using Cox proportional hazards for mortality, logistic regression for institutionalisation and falls, negative binomial regression for the number of secondary care 
contacts, and analysis of covariance for the EQ-5D score and log transformed General Health Questionnaire 12 score. We used logistic regression for binary variables created by dichotomising the activities of daily living and the ICECAP-O scores at the median value, as assumptions for analysis of covariance were not met. All models for the secondary outcomes included centre as a covariate; additional analyses also adjusted for pre-specified prognostically important covariates for each of these outcomes, chosen on the basis of the previous cohort study. ${ }^{3}$ However, additional analyses adjusting for baseline covariates were not possible for days at home (primary outcome) owing its irregular distribution.

Analyses for the secondary outcomes mortality, institutionalisation, and number of hospital presentations were adjusted for age, Identification of Seniors At Risk score, Charlson score, and Mini-Mental State Examination. The analysis for activities of daily living was adjusted for baseline activities of daily living, age, Charlson score, Identification of Seniors At Risk, and Mini-Mental State Examination. The analysis for psychological wellbeing was adjusted for baseline General Health Questionnaire, age, sex, activities of daily living, EQ-5D, and number of drugs. The quality of life analyses (EQ-5D and ICECAP-O) were adjusted for baseline EQ-5D/ICECAP-O, Identification of Seniors At Risk, activities of daily living, Mini-Mental State Examination, and number of drugs. The analysis for falls was adjusted for Identification of Seniors At Risk, Mini-Mental State Examination, and whether the participant presented with a fall at baseline. In addition, for the EQ-5D, General Health Questionnaire 12, activities of daily living, and falls outcomes, we used multiple imputation to explore the effect of missing data. We did a pre-planned subgroup analysis split by baseline Identification of Seniors At Risk score (2-3 v 4-6). We used Stata version 11 for all analyses, with labels for group allocation revealed after analyses were complete.

\section{Sample size}

Using pilot data in which the mean number of days spent at home at 90 days was 63 (SD 23), we calculated that a sample size of 200 in each group would have $90 \%$ power to detect a clinically important difference of 7.5 days in the mean number of days at home between the two groups at a 5\% significance level. To account for loss to follow-up, we set a total sample size of 420 participants.

\section{Results}

Participants were recruited from October 2010 until the end of February 2012. During this period, 1001 patients were identified as eligible for the study and 433 patients were recruited: 217 in the control group and 216 in the intervention group (figs $1 \Downarrow$ and $2 \Downarrow)$.

Table $1 \Downarrow$ shows the baseline characteristics of the two groups. One hundred and sixty nine participants did not have mental capacity to provide consent: 16 were recruited using a family consultee and 153 using a professional medical practitioner consultee. Baseline psychological wellbeing and self reported health status could not be collected for some participants recruited via a consultee, resulting in some missing data (table $1 \Downarrow)$.

Sixteen participants withdrew during the study: five in the control group and 11 in the intervention group. We did not include these participants in any of the analyses. We therefore included 212 control group participants and 205 intervention group participants in the analysis of the primary days at home outcome and the secondary outcomes for mortality, institutionalisation, and hospital presentations.

Table $2 \Downarrow$ shows a summary of the specialist geriatric medical intervention received by participants in the intervention groups. Nearly all $(201,98 \%)$ received the intervention as intended, and $133(66 \%)$ of these had a response beyond the initial assessment; 122 of these were seen at home a mean of 12 days after the initial assessment. Table $2 \Downarrow$ shows that the geriatricians took a range of actions, most commonly liaison with other practitioners, further diagnostic tests, drug changes, and referral for rehabilitation.

We found no difference in the primary outcome of the number of days spent at home between the two groups (mean 80.2 days in the control group and 79.7 in the intervention group; 95\% confidence interval for the difference in means -4.6 to 3.6 days; $\mathrm{P}=0.31$ ) (table $3 \Downarrow$ ). More than half of the participants spent all 90 days of the follow-up period at home (121/212 (57\%) in the control group and 106/205 (52\%) in the intervention group). Twenty six $(6 \%)$ participants died during the study, and nine (2\%) participants moved from the community to a permanent care home: we found no evidence of a difference between the two groups for these two outcomes. Two hundred and twenty six $(54 \%)$ participants had at least one hospital presentation during the study, with an increased number of hospital presentations in the intervention group (mean 0.94 hospital presentations during the 90 day follow-up period in the control group and 1.20 in the intervention group; $95 \%$ confidence interval for rate ratio 1.01 to $1.74 ; \mathrm{P}=0.05$ ).

Information from questionnaires for at least one of the activities of daily living, falls, health status, and psychological wellbeing outcomes was available for 313 participants at follow-up: 157 in the control group and 156 in the intervention group. The characteristics of the participants who completed and who did not complete the follow-up questionnaire were similar in both groups: participants who did not complete the questionnaire were slightly younger on average (mean 81.1 (SD 7.2) with no follow-up compared with 82.9 (6.6) with follow-up), were more often male $(47 \% v 35 \%)$, were more likely to have mental capacity at recruitment $(77 \% v 60 \%)$, more often lived in the community $(88 \% v 73 \%)$, and were more likely to have had a hospital presentation during the follow-up period (61\% v 50\%).

The baseline characteristics of the participants who completing the follow-up questionnaire were generally well matched, except for a greater proportion of participants in the control group presenting with reduced mobility as observed at baseline. Table $3 \Downarrow$ shows the number of participants who completed each of the secondary health outcomes and the estimates of the intervention effect for the complete cases: we found no evidence of a difference between the two treatment groups for any of these outcomes. The results from the analyses of the models including centre only and the models including the additional pre-specified prognostically important covariates were very similar, as were the results obtained using the multiply imputed data.

A sensitivity analysis (for the secondary outcomes) adjusting for presentation with reduced mobility, done because of the imbalance between the two groups at baseline, did not alter the results. The pre-planned subgroup analysis according to risk of adverse outcomes at recruitment (based on Identification of Seniors At Risk score 2 or $3 /$ score of 4 or more) showed no difference in the effect of the intervention for any of the outcomes. 


\section{Discussion}

In this study, the provision of specialist geriatric medical assessment and interventions led to no improvement in the clinical outcomes of at risk patients discharged from acute medical units.

Recruitment to target, adherence to the protocol, and the narrow confidence intervals for the primary outcome indicate that the results are robust and sufficiently precise. The findings of this sort of study depend on the relation between who was recruited, what was done to them, and what outcomes were measured. Although the Identification of Seniors At Risk score is the best evidenced tool for the purpose, ${ }^{22}$ its use in practice to identify high risk patients has proved to be less than ideal. ${ }^{35}$ A more accurate selection of high risk patients would have increased the potential size of the treatment effect. Notably, comorbidities were not common in this cohort (median one comorbidity per participant), although polypharmacy (median seven drugs per participant) and cognitive impairment (median Mini-Mental State Examination score 23) were present. A large proportion of eligible patients declined to give consent; if these had been high risk patients, their inclusion would similarly have increased the potential size of the treatment effect. The intervention delivered by the geriatricians was largely as intended and as might be delivered in routine practice, although much of the potential effect depended on the actions of others, such as when advice was given or referrals were made. The effects of the specialist geriatric input may have affected aspects of experience or outcome that were not used as trial outcomes, such as satisfaction with experience or outcome. Readmissions occurred in more than half of the participants within the three months' follow-up, so ample scope existed for an intervention to reduce readmissions and hence increase the amount of time participants spent at home. Even taking all these potential limitations into account, we can conclude that an intervention of this sort in a relatively high risk population had no notable effect on the measured outcomes.

The provision of specialist geriatric medical input was intended to overcome the inadequacies of medical care as previously identified, and we intended that liaison with other community staff and resources would enable the process of comprehensive geriatric assessment to be delivered. We believe that the most likely explanation for the lack of benefit of this intervention is that the specialist geriatric intervention tested in this study was, in effect, a liaison service and did not deliver comprehensive geriatric assessment. This explanation accords with the evidence base for comprehensive geriatric assessment. Comprehensive geriatric assessment, as a principle, is well known to lead to improved outcomes for frail older people. ${ }^{23-25}$ In particular, firm evidence supports acute geriatric units as a means of improving outcomes for older people in acute care, ${ }^{26}{ }^{27}$ but the evidence for liaison services is weak. ${ }^{28}{ }^{23} \mathrm{Few}$ previous studies have examined attempts to implement comprehensive geriatric assessment in this group of patients discharged from an acute assessment area of a hospital, ${ }^{6}$ although a recent report of a specialist frail older person unit in an English emergency department was promising. ${ }^{29}$

This study shows that simply providing isolated specialist geriatric input across the acute-community interface, to people identified as being at increased risk by using the Identification of Seniors At Risk score, is unlikely to have any measurable benefit in terms of patients' outcomes. Better methods of identifying patients who are likely to benefit from input need to be developed. Furthermore, given the wealth of information about the benefits of comprehensive geriatric assessment, improving the outcomes of frail older people discharged from acute assessment units is likely to require a more sophisticated, integrated intervention that enables the delivery of the comprehensive geriatric assessment process, such as the admission of such patients to "virtual wards." ${ }^{30}$ Given the failure of this intervention to show the desired effect, any such new interventions also have to be rigorously evaluated.

Janet Darby and the Trent and Leicestershire, Northamptonshire and Rutland Comprehensive Local Research Networks provided support for the recruitment of participants (Trent: Rachael Taylor, Caroline May, Diane Havard; Leicester and Northampton: Aidan Dunphy, Diane Galli). We acknowledge the help of IT services at Nottingham University Hospitals (Kate Moore, Samantha Cole), IT services in Leicester (lain Sands), and the patients who participated in the study. We also acknowledge the wider Medical Crises in Older People study group, which included Rowan Harwood, Anthony Avery, Sarah Lewis, Davina Porock, Rob Jones, Pip Logan, Justine Schneider, Jane Dyas, Adam Gordon, Sarah Goldberg, and Bella Robbins.

Contributors: JE contributed to the study design, conduct, and analysis. LB contributed to the analysis. MF was involved in the collection, methodology, and analysis of hospital resource use data. JG was the principal investigator and contributed to the study design, conduct, and analysis. SC had the initial idea for the study, is the grant holder, and contributed to the study design, conduct, and analysis. All authors contributed to the preparation of the manuscript. JG is the guarantor. Funding: This article presents independent research funded by the National Institute for Health Research (NIHR) under its Programme Grants for Applied Research funding scheme (RP-PG-0407-10147). The views expressed in this article are those of the authors and not necessarily those of the NHS, the NIHR, or the Department of Health. Competing interests: All authors have completed the ICMJE uniform disclosure form at www.icmje.org/coi_disclosure.pdf (available on request from the corresponding author) and declare: no support from any organisation for the submitted work; no financial relationships with any organisations that might have an interest in the submitted work in the previous three years; no other relationships or activities that could appear to have influenced the submitted work.

Ethical approval: Research ethics committee and regulatory approvals were obtained (Nottingham 1 Research Ethics Committee, reference 10/H0403/1).

Trial registration Current Controlled Trials ISRCTN21800480.

Data sharing: Technical appendix, statistical code, and dataset available from the corresponding author at spc3@le.ac.uk. Consent was not obtained for data sharing, but the presented data are anonymised and risk of identification is low.

1 Percival F, Day N, Lambourne A, Derek B, Ward D. An evaluation of consultant input into acute medical admissions management in England, Wales and Northern Ireland. Royal College of Physicians, 2010.

2 Woodard J, Gladman J, Conroy S. Frail older people at the interface. Age Ageing 2010;39(S1):i36

3 Edmans J, Bradshaw L, Gladman J, Franklin M, Berdunov V, Elliott RA, et al. The Identification of Seniors at Risk (ISAR) score to predict clinical outcomes and health service costs in older people discharged from UK acute medical units. Age Ageing 2013; published online 10 May.

4 Woodard J, Rowell G, Vartha R, Whittingham C, Vadher N, Conroy S. Appropriate prescribing in older people. J Nutr Health Aging 2009;13(suppl 1):S477.

5 Gladman J, Kearney F, Ali A, Blundell A, Wong R, Laithwaite E, et al. The role of the interface geriatrician across the acute medical unit/ community interface. Medical Crises in Older People. Discussion Paper Series Issue 9 February 2012 (available at http:// nottingham.ac.uk/mcop/documents/papers/issue9-mcop-issn2044-4230.pdf).

6 Conroy SP, Stevens T, Parker SG, Gladman JRF. A systematic review of comprehensive geriatric assessment to improve outcomes for frail older people being rapidly discharged from acute hospital: 'interface geriatrics'. Age Ageing 2011;40:436-43.

7 Conroy S, Stephens T, Gladman J. The interface between acute hospitals and community care for older people presenting to acute medical units: a mapping review. Medical Crises in Older People. Discussion Paper Series Issue 6 December 2010 (available at http:// www.nottingham.ac.uk/mcop/documents/papers/issue6-mcop-issn2044-4230.pdf).

8 Conroy S, Ferguson C, Banerjee J. Interface geriatrics: an evidence based solution for frail older people with medical crises. Br J Hosp Med 2010;71:98-101. 


\section{What is already known on this topic}

Most acute hospitals receive patients presenting as an emergency in an acute assessment unit Poor outcomes and high resource use are common in older people discharged to the community from acute assessment units Specialist geriatric medical intervention for at risk older people discharged to the community from acute assessment units may reduce the incidence of adverse outcomes and associated high resource use

\section{What this study adds}

Specialist geriatric medical intervention in an at risk population of older people discharged from acute assessment units had no effect on patient level outcomes or subsequent use of secondary care or long term care

Improving the outcomes of frail older people discharged from acute assessment units is likely to require a more sophisticated, integrated intervention that enables the delivery of the comprehensive geriatric assessment process

9 Gladman J, Donald I, Archard G, Morris J. Interface between primary and secondary medical care in the new NHS in England: the care of frail older people by GPs and consultant geriatricians, 2007. www.bgs.org.uk/index.php?option=com_content\& view=article\&id=373:interfaceprimarysecondaryscotland\&catid=59:communitycare \& Itemid $=554$.

10 Edmans J, Conroy S, Harwood R, Lewis S, Elliott R, Logan P, et al. Acute medical unit comprehensive geriatric assessment intervention study (AMIGOS): study protocol for a randomised controlled trial. Trials 2011;12:200.

11 McCusker J, Bellavance F, Cardin S, Trepanier S, Verdon J, Ardman O. Detection of older people at increased risk of adverse health outcomes after an emergency visit: the ISAR screening tool. J Am Geriatr Soc 1999;47:1229-37.

12 Dendukuri N, McCusker J, Belzile E. The identification of seniors at risk screening tool: further evidence of concurrent and predictive validity. J Am Geriatr Soc 2004;52:290-6.

13 McCusker J, Bellavance F, Cardin S, Belzile E, Verdon J. Prediction of hospital utilization among elderly patients during the 6 months after an emergency department visit. Ann Emerg Med 2000;36:438-45.

14 Cleves MA, Sanchez N, Draheim M. Evaluation of two competing methods for calculating Charlson's comorbidity index when analyzing short-term mortality using administrative data. J Clin Epidemiol 1997;50:903-8.

15 Folstein MF FS, McHugh PR. "Mini-mental state": a practical method for grading the cognitive state of patients for the clinician. J Psychiatr Res 1975;12:189-98

16 Dick JP, Guiloff RJ, Stewart A, Blackstock J, Bielawska C, Paul EA, et al. Mini-mental state examination in neurological patients. J Neurol Neurosurg Psychiatry 1984;47:496-9.

17 Wade DT, Collin C. The Barthel ADL index: a standard measure of physical disability? Int Disabil Stud 1988;10:64-7.

18 Brazier J. Using the SF-36 and Euroqol on an elderly population. Qual Life Res 1996;5:195-204

19 Coast J, Peters T, Natarajan L, Sproston K, Flynn T. An assessment of the construct validity of the descriptive system for the ICECAP capability measure for older people. Qual Life Res 2008;17:967-76.

20 Goldberg D, Hiller V. A scaled verison of the general health questionnaire. Phychol Med 1979;9:139-45

21 Gladman J, Harwood R, Conroy S, on behalf of the Medical Crises In Older People Study Group. Days at home: an outcome measure in studies of specialist services providing care for older people. Medical Crises in Older People. Discussion Paper Series Issue 4 October 2010 (available at http://nottingham.ac.uk/mcop/documents/papers/issue4-mcopissn2044-4230.pdf)

22 Edmans J, Gladman J, Havard D. Umbrella review of tools to assess risk of poor outcome in older people attending acute medical units. Medical Crises in Older People. Discussion
Paper Series Issue 11 June 2012 (available at http://nottingham.ac.uk/mcop/documents/ papers/issue11-mcop-issn2044-4230.pdf).

23 Ellis G, Whitehead MA, Robinson D, O'Neill D, Langhorne P. Comprehensive geriatric assessment for older adults admitted to hospital: meta-analysis of randomised controlled trials. BMJ 2011;343:d6553.

24 Beswick AD, Rees K, Dieppe P, Ayis S, Gooberman-Hill R, Horwood J, et al. Complex interventions to improve physical function and maintain independent living in elderly people: a systematic review and meta-analysis [See comment]. Lancet 2008;371:725-35.

25 Stuck AE, Siu AL, Wieland GD, Rubenstein LZ, Adams J. Comprehensive geriatric assessment: a meta-analysis of controlled trials. Lancet 1993;342:1032-6.

26 Baztan JJ, Suarez-Garcia FM, Lopez-Arrieta J, Rodriguez-Manas L, Rodriguez-Artalejo F. Effectiveness of acute geriatric units on functional decline, living at home, and case fatality among older patients admitted to hospital for acute medical disorders: meta-analysis. BMJ 2009;338:b50.

27 Fox MT, Persaud M, Maimets I, O'Brien K, Brooks D, Tregunno D, et al. Effectiveness of acute geriatric unit care using acute care for elders components: a systematic review and meta-analysis. J Am Geriatr Soc 2012;60:2237-45.

28 Deschodt M, Flamaing J, Haentjens P, Boonen S, Milisen K. Impact of geriatric consultation teams on clinical outcome in acute hospitals: a systematic review and meta-analysis. BMC Med 2013;11(1):48.

29 Conroy SP, Ansari K, Williams M, Laithwaite E, Teasdale B, Dawson J, et al. A controlled evaluation of comprehensive geriatric assessment in the emergency department: the 'Emergency Frailty Unit'. Age Ageing 2013; published online 23 July.

30 The Silver Book: Quality care for older people with urgent and emergency care needs. 2012. http://www2.le.ac.uk/departments/cardiovascular-sciences/people/conroy/the-silverbook/the-silver-book/.

Accepted: 03 September 2013

\section{Cite this as: BMJ 2013;347:f5874}

This is an Open Access article distributed in accordance with the Creative Commons Attribution Non Commercial (CC BY-NC 3.0) license, which permits others to distribute, remix, adapt, build upon this work non-commercially, and license their derivative works on different terms, provided the original work is properly cited and the use is non-commercial. See: http://creativecommons.org/licenses/by-nc/3.0/. 


\section{Tables}

Table 1| Baseline characteristics. Values are numbers (percentages) unless stated otherwise

\begin{tabular}{|c|c|c|c|}
\hline Characteristics & Control $(n=217)$ & Intervention ( $n=216$ ) & Overall $(n=433)$ \\
\hline \multicolumn{4}{|l|}{ Study centre: } \\
\hline Nottingham & $136(63)$ & $136(63)$ & $272(63)$ \\
\hline Leicester & $81(37)$ & $80(37)$ & $161(37)$ \\
\hline Mean (SD) age & $82.8(7.0)$ & $83.1(6.7)$ & $83.0(6.8)$ \\
\hline Female sex & $141(65)$ & $133(62)$ & $274(63)$ \\
\hline White ethnicity & $206(95)$ & $211(98)$ & $417(96)$ \\
\hline \multicolumn{4}{|l|}{ Residence at recruitment: } \\
\hline Alone & $90(41)$ & $85(39)$ & $175(40)$ \\
\hline With someone & $67(31)$ & $75(35)$ & $142(33)$ \\
\hline Care home & $60(28)$ & $56(26)$ & $116(27)$ \\
\hline Mental capacity to consent at recruitment & $131(60)$ & $133(62)$ & $264(61)$ \\
\hline Median (IQR) ISAR score & $3(3-4)$ & $3(2-4)$ & $3(3-4)$ \\
\hline Median (IQR) Charlson comorbidity score & $1(0-2)$ & $1(1-2)$ & $1(1-2)$ \\
\hline Median (IQR) No of drugs & $7(5-9)$ & $7(5-9)$ & $7(5-9)$ \\
\hline Presented with fall & $65(30)$ & $68(31)$ & $133(31)$ \\
\hline Presented with reduced mobility & $35(16)$ & $15(7)$ & $50(12)$ \\
\hline Presented with cognitive impairment/confusion & $26(12)$ & $42(19)$ & $68(16)$ \\
\hline Prior dementia diagnosis & $59(27)$ & $56(26)$ & $115(27)$ \\
\hline Cognitive function-median (IQR) MMSE & $23(12-26)$ & $23(11.5-27)$ & $23(12-26)$ \\
\hline Psychological wellbeing—median (IQR) GHQ12 & $11.5(8-15) ;(n=166)$ & $12(8-16) ;(n=162)$ & $12(8-15) ;(n=328)$ \\
\hline Activities of daily living_Barthel ADL $\geq 17$ & $100(51) ;(n=197)$ & $111(55) ;(n=202)$ & $211(53) ;(n=399)$ \\
\hline
\end{tabular}

GHQ12=General Health Questionnaire 12; IQR=interquartile range; ISAR=Identification of Seniors At Risk; MMSE=Mini-Mental State Examination. 
Table 2| Intervention summary

No $(\%)$ or mean (range)

Assessment and follow-up

\begin{tabular}{|c|c|}
\hline Allocated to intervention & 205 \\
\hline Received intervention as intended (initial assessment, home visit, clinic visit, phone call, other patient related activity) & $201(98)$ \\
\hline Received follow-up responses (home visit, clinic visit, phone call, other patient related activity) & $133(66)$ \\
\hline Initial assessment on ward $(\mathrm{n}=201)$ & $198(99)$ \\
\hline Initial assessment at home $(n=201)$ & $3(2)$ \\
\hline Interval from initial assessment to follow-up $(n=122)$ & $12(1-68)$ days \\
\hline Follow-up home visits & $87(43)$ \\
\hline Follow-up clinic visits & $13(7)$ \\
\hline Follow-up phone calls & $57(28)$ \\
\hline Other patient related activity & $98(49)$ \\
\hline Duration of initial assessments $(n=198)$ & $44.93(5-90) \min$ \\
\hline Duration of home visits (including travel) $(\mathrm{n}=87)$ & $76.17(30-120) \mathrm{min}$ \\
\hline Duration of follow-up phone calls $(n=57)$ & $15.02(2-60) \min$ \\
\hline Duration of other patient related activities $(n=98)$ & $22.82(5-150) \min$ \\
\hline Total geriatrician time per participant $(n=201)$ & $93.70(5-305) \mathrm{min}$ \\
\hline \multicolumn{2}{|l|}{ Specific interventions } \\
\hline Admission to hospital & $13(6)$ \\
\hline Change to drug treatment & $120(60)$ \\
\hline Advance care planning & $42(21)$ \\
\hline Liaison with other medical practitioners & $155(77)$ \\
\hline Health advice to patient & $66(33)$ \\
\hline \multicolumn{2}{|l|}{ Request for: } \\
\hline Further medical investigation & $57(28)$ \\
\hline Further medical treatment & $7(3)$ \\
\hline Additional medical follow-up & $52(26)$ \\
\hline \multicolumn{2}{|l|}{ Referral for: } \\
\hline Specialist nursing services & $28(14)$ \\
\hline Rehabilitation services & $58(29)$ \\
\hline Social care & $9(4)$ \\
\hline Other community services & $4(2)$ \\
\hline
\end{tabular}


Table 3| Outcomes at 90 days

\begin{tabular}{|c|c|c|c|}
\hline Outcome & Control $(n=217)$ & Intervention ( $n=216)$ & Intervention effect adjusted for centre \\
\hline No (\%) included in analysis at 90 days & $212(98)$ & $205(95)$ & - \\
\hline Mean (SD) days at home & $80.2(21.5)$ & $79.7(21.3)$ & $-0.5(-4.6$ to 3.6$) ; P=0.31$ \\
\hline No (\%) died (HR) & $12(6)$ & $14(7)$ & 1.22 (0.57 to 2.65$) ; P=0.61$ \\
\hline No (\%) institutionalisation (OR) & 4/156 (3) & $5 / 153(3)$ & 1.31 (0.34 to 4.97$) ; P=0.69$ \\
\hline Mean (SD) hospital presentations (RR) & $0.94(1.58)$ & $1.20(2.14)$ & 1.32 (1.01 to 1.74$) ; P=0.05$ \\
\hline No (\%) Barthel ADL $\geq 17$ (OR) & $67 / 157(43)$ & $75 / 156(48)$ & 1.25 (0.72 to 2.17$) ; P=0.42$ \\
\hline Geometric mean GHQ12 (ANCOVA) & $12.4(n=132)$ & $12.0(n=135)$ & 0.96 (0.87 to 1.06$) ; P=0.44$ \\
\hline Mean (SD) EQ-5D (ANCOVA) & $0.45(0.32) ;(n=139)$ & $0.45(0.32) ;(n=146)$ & $-0.01(-0.08$ to 0.06$) ; P=0.80$ \\
\hline No (\%) ICECAP-O $\geq 0.81(\mathrm{OR})$ & $54 / 120(45)$ & $72 / 131(55)$ & 1.38 ( 0.80 to 2.40$) ; P=0.25$ \\
\hline No (\%) self reported fall during follow-up (OR) & $66 / 155(43)$ & $64 / 156(41)$ & 0.94 (0.60 to 1.48 ); $P=0.79$ \\
\hline
\end{tabular}

$A D L=a c t i v i t i e s$ of daily living; ANCOVA=analysis of covariance; GHQ12=General Health Questionnaire 12; HR=hazard ratio; ICECAP-O=ICEpop CAPability measure for older people; $\mathrm{OR}=$ odds ratio; $\mathrm{RR}=$ rate ratio. 


\section{Figures}

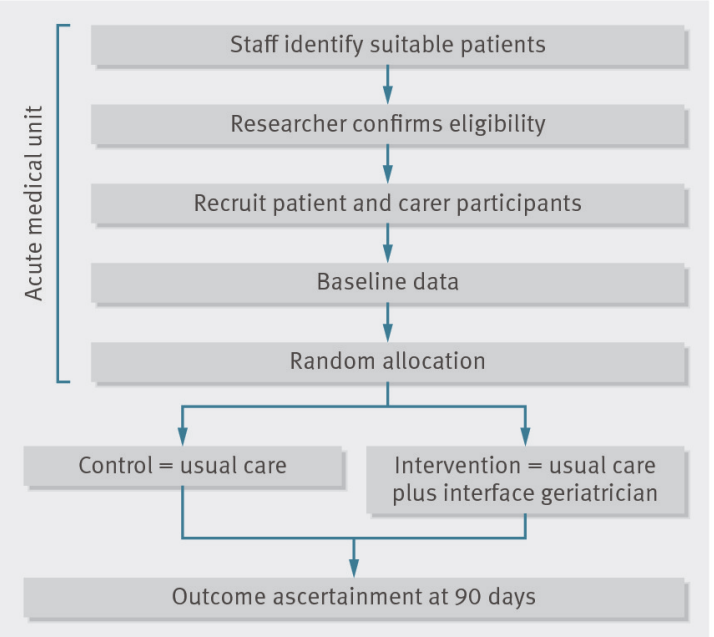

Fig 1 Overall outline of randomised controlled trial of comprehensive geriatric assessment intervention

\begin{tabular}{|c|c|}
\hline \multicolumn{2}{|c|}{ Assessed for eligibility ( $n=1001)$} \\
\hline & $\begin{array}{l}\text { Not included }(n=568): \\
\text { Excluded }(n=190) \\
\text { Declined consent }(n=378)\end{array}$ \\
\hline \multicolumn{2}{|c|}{ Randomised $(n=433)$} \\
\hline$\downarrow$ & \\
\hline $\begin{array}{l}\text { Allocated to control }(n=217) \text { : } \\
\text { Received allocated intervention }(n=216) \\
\text { Did not receive allocated intervention } \\
\text { (assessed by geriatrician) }(n=1)\end{array}$ & $\begin{array}{l}\text { Allocated to intervention }(n=216) \text { : } \\
\text { Received allocated intervention }(n=212) \\
\text { Did not receive allocated intervention } \\
\text { (not assessed by geriatrician) }(n=4)\end{array}$ \\
\hline$\downarrow$ & 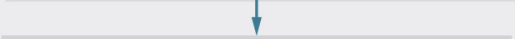 \\
\hline $\begin{array}{l}\text { Withdrawn at baseline after initial consent } \\
\text { from medical practitioner }(n=5) \\
\text { Dead at follow-up }(n=12) \\
\text { Withdrawn from clinical follow-up }(n=40) \\
\text { Died between follow-up date and } \\
\text { ascertainment }(n=3)\end{array}$ & $\begin{array}{l}\text { Withdrawn at baseline after initial consent } \\
\text { from medical practitioner }(n=11) \\
\text { Dead at follow-up }(n=14) \\
\text { Withdrawn from clinical follow-up }(n=34) \\
\text { Died between follow-up date and } \\
\text { ascertainment }(n=1)\end{array}$ \\
\hline$\gamma$ & 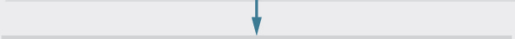 \\
\hline $\begin{array}{l}\text { Analysed for primary outcome and secondary } \\
\text { outcomes of mortality, institutionalisation, } \\
\text { and hospital presentations }(n=212)\end{array}$ & $\begin{array}{l}\text { Analysed for primary outcome and secondary } \\
\text { outcomes of mortality, institutionalisation, } \\
\text { and hospital presentations }(n=205)\end{array}$ \\
\hline $\begin{array}{l}\text { Questionnaire for other outcomes completed } \\
\text { at } 90 \text { days }(n=157) \text { : }\end{array}$ & $\begin{array}{l}\text { Questionnaire for other outcomes completed } \\
\text { at } 90 \text { days }(n=156) \text { : }\end{array}$ \\
\hline No/incomplete information on GHQ-12 $(n=25)$ & No/incomplete information on GHQ-12 $(n=21)$ \\
\hline No/incomplete information on EQ5D $(n=18)$ & No/incomplete information on EQ5D $(n=10)$ \\
\hline $\begin{array}{l}\text { No/incomplete information on ICECAP-O }(n=37) \\
\text { No information on falls }(n=2)\end{array}$ & No/incomplete information on ICECAP-O $(n=25)$ \\
\hline
\end{tabular}

Fig 2 Flow chart of study. GHQ=General Health Questionnaire; ICECAP-O=ICEpop CAPability measure for older people 